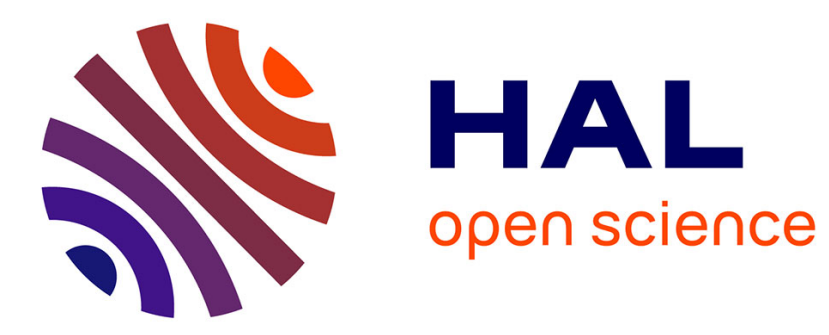

\title{
Les Méthodes Prédictives pour le Développement de Nouvelles Molécules Explosives
}

\author{
S. Dufort
}

\section{To cite this version:}

S. Dufort. Les Méthodes Prédictives pour le Développement de Nouvelles Molécules Explosives. Journal de Physique IV Proceedings, 1995, 05 (C4), pp.C4-303-C4-307. 10.1051/jp4:1995424 . jpa00253723

\section{HAL Id: jpa-00253723 \\ https://hal.science/jpa-00253723}

Submitted on 1 Jan 1995

HAL is a multi-disciplinary open access archive for the deposit and dissemination of scientific research documents, whether they are published or not. The documents may come from teaching and research institutions in France or abroad, or from public or private research centers.
L'archive ouverte pluridisciplinaire HAL, est destinée au dépôt et à la diffusion de documents scientifiques de niveau recherche, publiés ou non, émanant des établissements d'enseignement et de recherche français ou étrangers, des laboratoires publics ou privés. 


\title{
Les Méthodes Prédictives pour le Développement de Nouvelles Molécules Explosives
}

\author{
S. Dufort
}

Commissariat à l'Energie Atomique, Centre d'Etudes du Ripault, BP. 16, 37260 Monts, France

L'utilisation de méthodes prédictives permettant de juger, avant synthèse, des performances et de la sécurité d'un nouvel explosif constitue aujourd'hui un élément important dans l'organisation des travaux des laboratoires de chimie des matériaux énergétiques. L'emploi de telles méthodes est rendu d'autant plus nécessaire que les cahiers des charges apparaissent de plus en plus exigeants, tant sur le plan des propriétés physiques du matériau que sur celui du coût de la molécule. Dans ces conditions, la mise au point d'un nouvel explosif satisfaisant l'ensemble des contraintes ne peut plus résulter du seul talent du chimiste organicien, mais doit s'appuyer sur une véritable démarche d'ingénierie moléculaire intégrant les paramètres physiques, chimiques et économiques du problème.

Les études conduisant à l'établissement d'un outil prédictif peuvent se classer en deux grandes catégories :

- les méthodes fondées sur la recherche de corrélations empiriques par l'analyse statistique de molécules connues : c'est le cas, par exemple, de la méthode de Rothstein et Petersen pour l'estimation des pression et vitesse de détonation d'un explosif à partir de sa seule formule moléculaire brute,

- les méthodes issues de l'étude physique du comportement de la molécule soumise à une sollicitation, dans le but de comprendre les relations entre la structure du motif chimique et ses propriétés expérimentales.

C'est cette seconde voie qui a été développée, depuis près de vingt ans, par le Commissariat à l' Energie Atomique. Ce choix ambitieux est étayé par la mauvaise fiabilité des méthodes empiriques lorsqu'elles s'adressent à des molécules autres que celles qui ont servi à leur établissement. Nous présentons dans cet exposé le principe des travaux effectués, leur retombée en termes de conception de nouveaux explosifs et les perspectives offertes par les progrès des techniques théoriques et expérimentales. 


\section{$\underline{\text { I-Les bases physiques des méthodes prédictives développées au C.E.A. }}$}

a) Structure chimique et caractéristiques de sécurité des explosifs (sensibilité au choc et stabilité thermique)

Afin d'approcher à l'échelle moléculaire le comportement de l'explosif soumis à une sollicitation par choc, nous nous sommes intéressés, du double point de vue expérimental et théorique, aux modifications d'ordre structural subies par la molécule juste derrière le passage du front de choc et avant décomposition. Le recueil de ces informations, sans perturber le milieu observé, a nécessité la mise au point d'un montage de spectrométrie à haute résolution temporelle. Ce montage utilise la diffusion Raman de la lumière ; il permet, en un point bien localisé de l'explosif et dans un intervalle de temps très bref ( 10 ns ), d'enregistrer les spectres de vibration caractéristiques de l'état de la molécule à l'instant de l'analyse. La comparaison des spectres obtenus au repos et derrière l'onde de choc renseigne sur la nature et l'ampleur des modifications apportées à la structure chimique de l'explosif. La figure ci-dessous illustre, à titre d'exemple, un résultat-type relatif aux bandes de vibration $\mathrm{N}-\mathrm{NO}_{2}$ de l'hexogène:
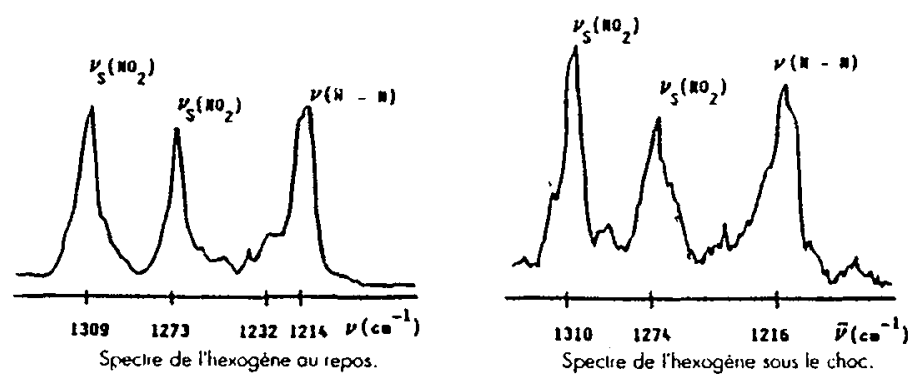

De façon générale, et durant les 50 ns séparant le passage du choc du début de la décomposition chimique, les spectres obtenus se caractérisent par

- des fréquences de raies peu perturbées, montrant que la géométrie de la molécule reste identique sous choc,

- d'importantes fluctuations de l'intensité des bandes, traduisant une profonde modification de la structure électronique de l'explosif.

Ces observations sont à la base des développements théoriques menés en parallèle autour de l'analyse de l'influence de la structure électronique d'une molécule explosive sur sa sensibilité au choc. Il s'agissait :

- d'une part, de comprendre et de modéliser les mécanismes d'interaction onde de choc molécule,

- d'autre part, de dégager les paramètres caractéristiques de la structure électronique d'un explosif représentatifs de sa sensibilité au choc expérimentale. 
Les méthodes de la Chimie Théorique nous ont permis d'amener des réponses à ces interrogations. Tout d'abord en assimilant le choc, à l'échelle microscopique, à une onde de compression entraînant sur son passage une diminution des distances inter moléculaires et, par suite, une brusque variation des potentiels électrostatiques entre molécules. Le modèle d'interaction onde de choc - molécule se ramène alors à la perturbation d'un objet quantique (la molécule) par un front de potentiel voyageant à la vitesse du choc. Le développement par la Mécanique Quantique de ce principe a montré l'existence d'un processus non uniforme d'excitation à des énergies élevées, de l'ordre de quelques $\mathrm{eV}$. Ce résultat, tout à fait original, conforte l'idée d'un mécanisme d'excitation conduisant à des niveaux électroniques spécialisés de la molécule.

D'autre part, l'analyse détaillée des états électroniques d'une soixantaine d'explosifs organiques a montré l'existence d'une forte corrélation entre l'évolution de la polarité de la liaison explosophore au cours de l'excitation et l'échelle expérimentale des sensibilités au choc. Cette relation, proposée par A. DELPUECH dès 1977, est à la base des calculs prédictifs effectués aujourd'hui afin d'évaluer la sensibilité des explosifs étudiés.

Une approche similaire a également été mise en oeuvre afin d'interpréter la réponse moléculaire aux sollicitations d'origine thermique. Dans ce cas, ce sont des mécanismes faisant intervenir l'état électronique fondamental qui sont pris en compte; la plus faible énergie d'activation de dissociation d'une liaison chimique associée à cet état permet d'apprécier a priori la température de décomposition d'une nouvelle molécule.

\section{b) Caractéristiques énergétiques des explosifs.}

Le deuxième grand volet de notre méthodologie prédictive se situe au niveau des propriétés énergétiques de la molécule. L'évaluation de ces propriétés a nécessité la mise au point d'un outil numérique performant, le code ETARC ( Equilibre Thermodynamique Avec Réactions Chimiques ). Ce code permet de proposer une description physique des produits de détonation à partir de la seule connaissance de la composition chimique de la molécule, de sa densité et de son enthalpie de formation. L'utilisation de ce logiciel a pour objectif de prévoir, outre les paramètres classiques pression et vitesse de détonation, l'énergie transmise au milieu connexe par l'expansion des produits de détonation.

\section{II-Du prédictif à l'ingénierie moléculaire}

L'ensemble de la démarche prédictive mise en oeuvre au CEA est rassemblée dans l'encadré 1. On remarquera, en plus des méthodes associées aux caractéristiques physiques de l'explosif (énergie et sécurité), le recours à un système expert de synthèse assistée par ordinateur ( SESAME) chargé d'appréhender les grandes voies chimiques applicables à la synthèse d'une nouvelle molécule, compte tenu de sa structure. Ces informations, préliminaires à la décision de développement du nouvel explosif, constituent un élément fondamental pour juger de la complexité du procédé de synthèse (donc de son coût) et de sa capacité à supporter un parcours ultérieur d'industrialisation. 
Quelle que soit l'efficacité des méthodes prédictives, la question importante reste malgré tout de savoir concevoir sur le papier un motif moléculaire en mesure de satisfaire les exigences de cahiers des charges très contraignants. La disponibilité de modèles établis par l'analyse approfondie du comportement des explosifs et l'expérience de l'application des outils qui en découlent à un grand nombre de molécules, débouche sur la compréhension du rôle joué par les différents groupements chimiques composant un édifice moléculaire. Il en résulte de véritables "règles de construction", que nous illustrerons par l'exemple du DANTNP. Cette molécule devait répondre à trois objectifs :

- un objectif de thermostatibilité (température de décomposition $>300^{\circ} \mathrm{C}$ ),

- un objectif de sensibilité au choc $(\mathrm{H} 50 \%(5 \mathrm{~kg})>60 \mathrm{~cm})$,

- un objectif énergétique : gain de $20 \%$ par rapport au TATB.

La conception du DANTNP repose sur deux étapes d'ingénierie moléculaire :

1. Mise en évidence par les études de Mécanique Quantique de l'intérêt du motif aminonitro-triazole (ANT) comme générateur d'insensibilité,

2. Greffage de deux de ces motifs sur un cycle thermostable afin d'assurer l'énergie et la tenue thermique du matériau.

La synthèse du DANTNP a été réalisée et une composition explosive élaborée. Ses propriétés, prévisionnelles et expérimentales, sont regroupées dans l'encadré 2.

\section{III - Perspectives}

Cette présentation dresse l'état des connaissances acquises dans nos laboratoires; si elles se sont d'ores et déjà traduites par de grands progrès dans la rationalisation de nos recherches, des points particuliers doivent encore être éclaircis :

- au niveau de la densité, dont la prédiction par des méthodes empiriques conduit bien souvent à des valeurs éloignées de la réalité. Les progrès observés dans le domaine de la Mécanique Moléculaire devraient permettre d'améliorer les choses, grâce à une capacité accrue à prendre en compte des systèmes polymoléculaires simulant l'état solide.

- au niveau de la manière dont l'explosif délivre son énergie dans le temps, paramètre sans doute plus important que la vitesse de détonation pour le dimensionnement des édifices pyrotechniques. Le développement de nouvelles méthodes spectrométriques aptes à identifier les mécanismes chimiques accompagnant la décomposition est un maillon essentiel pour la modélisation du processus de détente des produits de détonation.

Ces deux points font actuellement l'objet de recherches actives au CEA 
CONCEPTION ET SYNTHESE DE NOUVELLES MOLECULES EXPLOSIVES

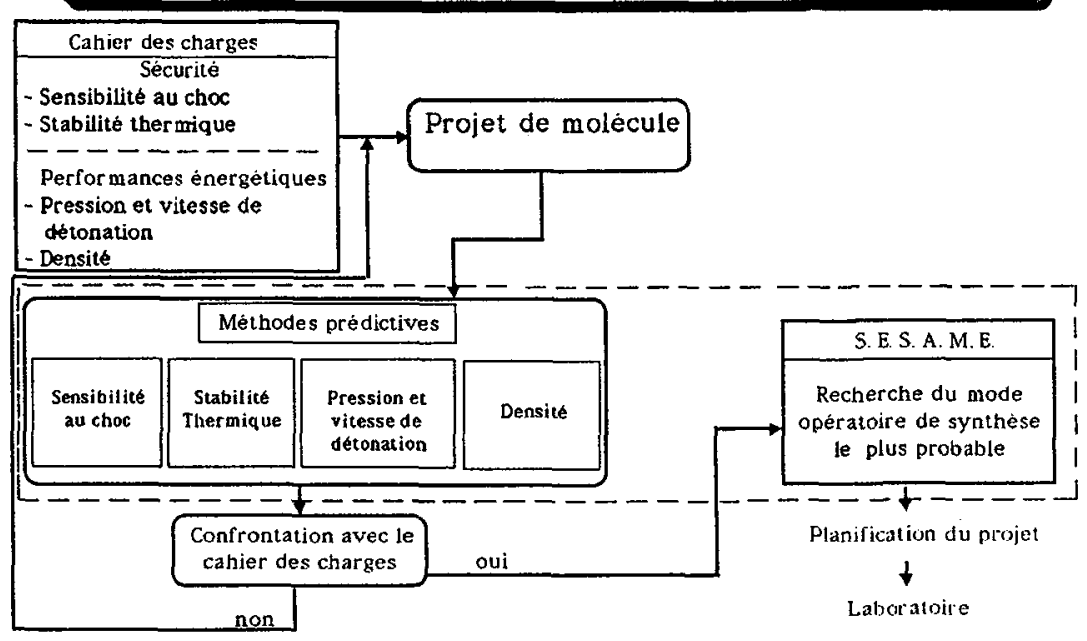

\begin{tabular}{|l|c|c|c|}
\cline { 2 - 4 } \multicolumn{1}{l|}{} & Objoctf & Prédictf & Exporimental \\
\hline Sensibilité au choc $(\mathrm{s} \times \mathrm{g})-\mathrm{cm}$ & $>60$ & $60-70$ & 70 \\
\hline Thermostabillte & $>300$ & $>300$ & 350 \\
\hline PCJ & $>330$ & 330 & 327 \\
\hline Densité & & 1,85 & 1,865 \\
\hline Vitesse do dótonation $(\mathrm{m} / \mathrm{s})$ & & $8100-8200$ & 8200 \\
\hline W/W TATB & $+20 \%$ & $+18 \%$ & $+15 \%$ \\
\hline
\end{tabular}<smiles></smiles> 